\title{
HARMONIC ANALYSIS ON SYMMETRIC STEIN MANIFOLDS FROM THE POINT OF VIEW OF COMPLEX ANALYSIS
}

\author{
SimON Gindikin
}

\begin{abstract}
The classical theory of finite dimensional representations of compact and complex semisimple Lie groups is discussed from the perspective of multidimensional complex geometry and analysis. The key tool is the complex horospherical transform which establishes a duality between spaces of holomorphic functions on symmetric Stein manifolds and dual horospherical manifolds.
\end{abstract}

Almost 50 years ago I.Gelfand suggested that the conception of the horospherical transform is the universal way to solve problems of harmonic analysis on homogeneous spaces. This philosophy replaces operating with groups by way of working with some geometrical structures which exist for a broad class of manifolds not only homogeneous ones. Eventually, theory of representations would be transformed into a much more general subject of geometric analysis where groups are not a necessary ingredient. Gelfand and Graev gave several remarkable examples of the realization of this idea (complex semisimple groups, Riemann symmetric manifolds, a few pseudo Riemann symmetric and non symmetric manifolds). Simultaneously, it was clear that the method of horospheres (at least in the initial form) does not work in such elementary situations as the group $\mathrm{SL}(2, \mathbb{R})$. Roughly speaking, this method does not work outside of the most continuous spectrum: discrete series of representations lie in the kernel of the horospherical transform.

Several years ago I suggested a way to solve this contradiction, at least in some cases, using a complex version of horospherical transform on real symmetric spaces: in a sense, we replace $\delta$-functions on the Cauchy kernels. This idea works for $\mathrm{SL}(2, \mathbb{R})[\mathrm{Gi00,Gi00}]$. In [GKO05] this was realized for arbitrary holomorphic discrete series. Deliberating upon the general context of complex horospherical transform, I found [Gi04,Gi05, Gi05'] with a surprise that this method works for compact Lie groups and, more generally, for compact symmetric spaces where real horospheres do not exist at all. It turned out, that compact symmetric spaces (starting from the real sphere) have canonical dual objects which are complex manifolds. In such a situation it is natural to understand this picture more systematically from the point of view of complex analysis. This is the aim of this paper. We believe that these considerations add something new to the entirely classical subject of finite dimensional representations and delivers some new observations in multidimensional 
complex analysis as well. It maybe that some interesting possibilities were missed in the beginning of noncommutative harmonic analysis.

Principal geometrical objects. We are interested, at first, in analysis on complex semisimple Lie groups, but it is natural to consider a more general class of objects - symmetric Stein manifolds with complex groups of automorphisms. They all have the structure $Z=G / H$ where $G$ is a complex semisimple Lie group and $H$ is its subgroup separated by a holomorphic involution. There are also Hermitian symmetric spaces of noncompact type which are also Stein manifolds, but their groups of automorphisms are not complex and we do not consider this case here.

Any complex semisimple group $G_{1}$ is a symmetric Stein manifold relative to the action of $G=G_{1} \times G_{1}$ and the diagonal subgroup $H=G_{1}$. We can consider for example, the group $Z_{0}=\operatorname{SL}(n, \mathbb{C})$. Let us give a few other examples. The complex hyperboloid (sphere) $Z_{1}$ is defined in $\mathbb{C}^{n+1}$ by the equation

$$
\square(z)=\left(z_{1}\right)^{2}+\cdots+\left(z_{n+1}\right)^{2}=1 .
$$

It is a Stein symmetric manifold with the group $G=\operatorname{SO}(n+1, \mathbb{C})$. If $G=\operatorname{SL}(2, \mathbb{C})$ and $H$ is the diagonal subgroup, then $G / H$ can be realized as the manifold of pairs of different points of the projective line $\mathbb{C} P^{1}$. A more general example $Z_{2}=$ $\mathrm{SL}(2 n) / \mathrm{S}(\mathrm{L}(\mathrm{n}) \times \mathrm{L}(\mathrm{n}))$ is realized as the manifold of generic pairs of $(n-1)$-planes in $\mathbb{C} P^{2 n-1}$. The symmetric Stein manifold $Z_{3}=\mathrm{SL}(n, \mathbb{C}) / \mathrm{SO}(n, \mathbb{C})$ can be realized as the manifold of complex symmetric matrices with the determinant 1 .

Let $A$ be the maximal Abelian subgroup in $G$ transversal to $H$ (Cartanian subgroup of $Z$ ) and $N$ be a corresponding unipotent subgroup such that

$$
G^{0}=H A N
$$

is a dense (Zariski open) subset of $G$ (complex Iwasawa decomposition). It is the holomorphic extension of the Iwasawa decomposition of a real form $G_{\mathbb{R}}$ of $G$ which has the maximal compact intersection with $H$. Let $n=\operatorname{dim} Z$ and $l=\operatorname{dim} A$ - the rank of the symmetric manifold $Z$.

Let $M$ be the centralizer of $A$ in $H$. Then the flag manifold

$$
F=G / A M N
$$

( $P=A M N$ is a parabolic subgroup) is considered as a standard object dual to the symmetric space $Z$. We prefer to work with a slightly different homogeneous space. Let us call

$$
\Xi=G / M N
$$

the horospheric manifold. There is a natural fibering $\Xi \rightarrow F$ with the fibers isomorphic to $A$.

Let us remark that manifolds $Z$ and $\Xi$ have the same dimension. Since the flag manifold $F$ is compact there are only constant holomorphic functions on $F$. The horospherical manifolds $\Xi$ are not Stein manifolds but there are a lot of holomorphic functions on them: the condition of holomorphic separation is satisfied. Our first 
principal aim is the construction of a duality between holomorphic functions on $Z$ and $\Xi$.

For the hyperboloid $Z_{1}$ the horospherical space $\Xi_{1}$ is the cone $\square(\zeta)=0, \zeta \neq 0$, in $\mathbb{C}_{\zeta}^{n+1}$; its projectivization - the quadric in $\mathbb{C} P^{n}$ - is the corresponding flag manifold $F_{1}$. For the manifold of unimodular symmetric matrices $Z_{3}$ the flag manifold $F_{3}$ is the manifold of complete flags in $\mathbb{C} P^{n-1}$ and the horospheric manifold $\Xi_{3}$ is the manifold of affine flags in $\mathbb{C}^{n}$ which are in the general position with a fixed "zero" flag.

If $Z$ is a semisimple group $G_{1}$ then $M N$ is the subgroup in $G=G_{1} \times G_{1}$ which is the product of 2 Borelian subgroups with a joint Cartanian subgroup $A_{1} \subset G_{1}$ (this subgroup is the centralizer of $N_{1} \times N_{1}$ in the diagonal embedding of $G_{1}$; here $N_{1}$ is a maximal unipotent subgroup of $G_{1}$ ).

Using the standard language of dual fiberings $Z \leftarrow G \rightarrow \Xi$ we can construct the geometrical duality between $Z$ and $\Xi$. Let $H$ and $M N$ be subgroups of $G$ in the complex Iwasawa decomposition. We realize points $z \in Z$ as classes $\{H g\}$ and points $\zeta \in \Xi$ as classes $\{g M N\}$ in the group $G$. Correspondingly, we connect the action of $G$ with the right multiplications of classes: $z \mapsto z \cdot g$ for classes $z \in Z$ and the left multiplications $\zeta \mapsto g \cdot \zeta$ for classes $\zeta \in \Xi$.

We take $M N$-classes in $G$ corresponding to points $\zeta \in \Xi$ and their projections on $Z$. The images $E(\zeta)$ are called the horospheres on $Z$. Their codimension is equal $\operatorname{rank} Z=\operatorname{dim} A=l: \operatorname{dim} E(\zeta)=n-l$. They are nondegenerate orbits of $N$ and of its conjugated subgroups. Sometimes we fix the"initial" horosphere $E\left(\zeta_{0}\right)$ corresponding to the subgroup $M N$ itself. Correspondingly, to points $z \in Z$ correspond the submanifolds $\Pi(z)$ which we will call the pseudospheres on $\Xi$. They are nondegenerate orbits of subgroups conjugated to $H$ and they are isomorphic to the homogeneous Stein manifold $H / M$.

Let us look at the simplest example of the hyperboloid $Z_{1}$. Let $\langle\zeta, z\rangle$ be the bilinear form corresponding to the quadratic form $\square(z)$. Then the horospheres $E(\zeta)$ are the paraboloids - the intersections of the hyperboloid by the isotropic hyperplanes

$$
\langle\zeta, z\rangle=1, \quad \square(\zeta)=0 .
$$

The pseudospheres $\Pi(z)$ are hyperboloids - the intersections of the cone $\Xi_{1}$ by the hyperplanes

$$
\langle\zeta, z\rangle=1, \quad \square(z)=1 .
$$

Spherical finite dimensional representations. Let us consider finite dimensional irreducible representations of $G$ which are realized in holomorphic functions on $Z$ and call them spherical (finite dimensional) representations on $Z$. Originally they were considered on compact symmetric spaces $X$ which are compact forms of $Z$, but these representations admit the holomorphic extension on $Z$ (unitary trick of H.Weyl); it is a classical object. In the case of the hyperboloid the compact space $X$ is the sphere and we have the representations in spherical polynomials.

Let us call finite dimensional irreducible representations of $G$ in holomorphic functions on $\Xi$ horospherical finite dimensional representations. The central fact is that the sets of spherical and horospherical irreducible representations coincide. It 
is a very deep fact: in one direction it means existence of the highest weight vectors in spherical representations; in another direction - the existence of zonal spherical functions in the horospherical representations. We want to replace these algebraic considerations of individual representations by a global fact of complex analysis.

Let us make a remark about the horospherical representations. The action $G$ on $\Xi$ corresponds to left multiplications on elements $g \in G$. It is remarkable that the right multiplications on $A$ are also well defined on classes $\{g M N\}$ :

$$
\zeta \mapsto \zeta \cdot a, \zeta \in \Xi, a \in A
$$

This operator of "right $A$-multiplication" $R(a)$, which commutates with the "left" action of the group $G$, plays an exceptional role in analysis on $\Xi$ and is the most important advantage of working with the horospherical manifold. Orbits of $R(A)$ are fibers of the fibering $\Xi \rightarrow F$. Pseudospheres $\Pi(z)$ are transversal to fibers and they intersect almost all fibers (corresponding to a Zariski open $H$-orbit on $F$ ).

For any holomorphic character $\lambda$ of the Abelian group $A$ let $\mathcal{O}_{\lambda}(\Xi)$ be the space of such holomorphic functions on $\Xi$ that

$$
f(\zeta \cdot a)=\lambda(a) f(\zeta), \quad a \in A, \zeta \in \Xi
$$

These spaces are $G$-invariant and the horospherical irreducible representations are realized in these $G$-modules. Using the fibering of $\Xi$ over the flag manifold $F$ with the fiber $A$ we can interpret $\mathcal{O}_{\lambda}(\Xi)$ as spaces of sections of line bundles on $F$. This elegant form of the theory of highest weights is the Borel-Weil theorem.

Let $\mathcal{O}(\Xi)$ (correspondingly $\mathcal{O}(Z)$ ) be the space of all holomorphic functions on $\Xi)$ (correspondingly on $Z$ ). We have the decomposition

$$
\mathcal{O}(\Xi)=\bigoplus \mathcal{O}_{\lambda}(\Xi)
$$

where we take the convergency on compacts in $\Xi$. To see this we can take a maximal torus $T \subset A$ and consider the decomposition in Fourier series on $T$-orbits. It is important that we do not need any $L^{2}$-structure for the decomposition $\mathcal{O}(\Xi)$ on irreducible modules.

Our first principal point is the analytic nature of the coincidence of sets of spherical and horospherical representations.

Theorem 1. The spaces of holomorphic functions $\mathcal{O}(Z)$ and $\mathcal{O}(\Xi)$ are isomorphic as $G$-modules.

Of course, the coincidence of spectrums of irreducible spherical and horospherical representations is the corollary of this theorem, but the equivalency of these reducible infinite-dimensional nonunitary representations is the stronger fact. It has an analytical nature. Let us emphasize that the manifolds $Z$ and $\Xi$ are not biholomorphically equivalent (as homogeneous manifolds with the same group $G$ and with nonisomorphic isotropy subgroups). Also the Stein extension of $\Xi$ is singular. 
Some preliminary constructions. To prove this Theorem we construct some intertwining operators between these spaces of holomorphic functions which in a natural sense are singular integral operators between holomorphic functions. The construction follows to the conception of the horospherical transform, but its realization in the holomorphic situation requiers several serious clarifications. These preparations have 2 components. Our operators will be Cauchy type operators with singularities on horospheres, and so we need to prepare these kernels; then we also need to select an appropriate collection of cycles for the integration which will avoid the singularities of the kernels. It is especially non trivial when the codimension of horospheres $l$ (the rank of $Z$ ) is more than 1 .

The characters $\lambda$ of $A$ which correspond to irreducible horospherical representations admit the next description [H94]. We describe them through highest weights. Let $\mathfrak{a}$ be the Lie algebra of the Abelian group $A$ and $\mathfrak{a}=\mathfrak{a}_{r}+i \mathfrak{a}_{i}$ is its orthogonal decomposition such that the torus $T=\exp \left(i \mathfrak{a}_{i}\right)$ and $A_{r}=\exp \left(\mathfrak{a}_{r}\right)$ is a totaly noncompact form of $A$. Let $\Sigma_{+}$be a system of positive (restricted) roots, corresponding to $\mathfrak{a}_{r}$ and $\left(\beta_{1}, \ldots \beta_{l}\right)$ be a basis of unmultiple roots. Let $\left(\mu_{1}, \ldots, \mu_{l}\right)$ be the dual system of weights:

$$
\frac{\left\langle\mu_{i}, \beta_{j}\right\rangle}{\left\langle\beta_{j}, \beta_{j}\right\rangle}=\delta_{i j}
$$

Then

$$
\Lambda=\left\{\mu=m_{1} \mu_{1}+\cdots+m_{l} \mu_{l} ; m_{j} \in \mathbb{Z}_{+}\right\}
$$

is the set of highest (restricted) weights of spherical (finite-dimensional) representations corresponding to the symmetric space $Z$ (or its compact form). The corresponding characters are

$$
a^{\mu}=\exp (\mu(\log (a))), \quad a \in A .
$$

Thus components in the decomposition of $\mathcal{O}(\Xi)$ are parameterized by $\Lambda \cong\left(\mathbb{Z}_{+}\right)^{l}$.

We use these characters for the definition of principal special functions on $Z$. For $z \in G^{0}$ let $a(z)$ be the Iwasawa projection on $A$. Let us call Silvester's functions on $Z$ the functions

$$
\Delta_{j}(z)=a(z)^{\mu_{j}}, \quad 1 \leq j \leq l .
$$

These functions (as all spherical functions of irreducible spherical representations), initially defined on the Zariski open part of $Z$ (corresponding to $G^{0}$ ) holomorphically extend on the whole $Z$. The origin of their name is that for the space $Z_{3}$ of symmetric unimodular matrices the functions $\Delta_{j}(z)$ are the principal upper minors (if $N=N_{+}$is the upper triangle group) which participate in the classical Silvester condition. If $Z_{0}=G_{1}=\operatorname{SL}(n, \mathbb{C})$ then the Silvester's functions are also upper principal minors if $N=\left(N_{-}, N_{+}\right) \subset G=G_{1} \times G_{1}$.

Silvester's functions play for general $Z$ the role similar to that the principal minors play in the classical Silvester condition. The set $G^{0}$ where the Iwasawa decomposition exists is defined by the conditions

$$
\Delta_{j}(z) \neq 0, \quad 1 \leq j \leq l .
$$


The equations $\Delta_{j}(z)=1, \quad 1 \leq j \leq l$, define the "initial" horosphere $E\left(\zeta_{0}\right)$ corresponding to the embedding of the unipotent group $N$ in $Z$. To receive the similar equations for other horospheres let us define

$$
\Delta_{j}(z \mid \zeta)=\Delta_{j}(z g)
$$

where $g$ is any representative of the class $\zeta=\left\{M N g_{0}\right\}$. Let us remark that $\Delta_{j}(z n)=\Delta_{j}(z), n \in N$. We then define the horosphere $E(\zeta), \zeta \in \Xi$, by the system of holomorphic equations

$$
\Delta_{j}(z \mid \zeta)=1, \quad 1 \leq j \leq l .
$$

Let us remark that in the simplest case of the hyperboloid $Z_{1}$ the rank (the codimension of horospheres) is equal 1 and there is only one Silvester's function $\Delta(z \mid \zeta)=\langle z, \zeta\rangle$.

As the set $V$ of cycles $X(v)$ we take the set of all compact forms of the Stein symmetric manifold $Z$. Let us take the maximal compact subgroup $U$ in $G$ such that the involution defining $U$ preserves $H$. Then

$$
X(0)=U /(U \cap H)
$$

is a compact symmetric space which is a real form of $Z$ and suppose that the torus $T \subset U$. By the action of elements $G$ we receive other compact forms $X(v)$ which we can parameterize by points $v \in V=G / U$. They are mutually homological and are totally real cycles of maximal dimension (their real dimension is equal to complex dimension of $Z$ ).

If $Z$ is a group then cycles $X(v)$ are its compact forms. For the hyperboloid $Z_{1}$ the cycles $X(v)$ are real spheres; as $X(0)$ we can take the sphere

$$
\left(x_{1}\right)^{2}+\cdots+\left(x_{n+1}\right)^{2}=1 \text {. }
$$

For the manifold $Z_{3}$ of unimodular symmetric matrices we can take as $X(0)$ the real submanifold of unitary symmetric matices; this compact manifold is symmetric relative to the action of the unitary group. In the example of $Z_{2}$ the compact form is the Grassmanian $\operatorname{Gr}_{\mathbb{C}}(n, 2 n)$ of $(n-1)$-planes in $\mathbb{C} P^{2 n-1}$.

The possibility to well define the intertwining operator follows from the next important Lemma due Clerc [Cl88]:

Lemma. We have

$$
\Delta_{j}(z) \leq 1, \quad 1 \leq j \leq l
$$

for $z \in X(0)$.

For our choice of $X(0)$ the intersection $X(0) \cap A$ is the maximal torus $T$ in $A$ which is defined by the conditions $\left|\Delta_{j}(z)\right|=1, \quad 1 \leq j \leq l$. Let us define

$$
\Xi(0)=\left\{\zeta \in \Xi ; \quad \Delta_{j}(x \mid \zeta)<1 \quad \text { for all } \quad z \in X(0) \text { and } j\right\}
$$


It is a domain in $\Xi$ of the next structure. We have

$$
\Delta_{j}(z a)=a^{\mu_{j}} \Delta_{j}(z), a \in A .
$$

As we mentioned, the horospherical manifold $\Xi$ fibers over the flag manifold $F$ on the orbits of the right action of $A$. The fibers correspond to the families of parallel horospheres on $Z$ - orbits of the same unipotent subgroup. Let us take the fiber through the initial horosphere $E\left(\zeta_{0}\right)$. It has the natural $A$-parametrization, induced by the Iwasawa decomposition: $\zeta=\zeta_{0} \cdot a$. It follows from the Lemma that the intersection $A_{+}$of this fiber with $\Xi(0)$ is defined in $A$ by the conditions

$$
\left|a^{\mu_{j}}\right|<1, \quad 1 \leq j \leq l
$$

so the torus $T$ is the edge of this polydisc.

The compact group $U$ acts transitively on both compact symmetric space $X(0)$ and the flag manifold $F(F=U / T(M \cap U))$. Therefore the action of $U$ translates the polydisc $A_{0}$ in the intersections of the domain $\Xi(0)$ with other fibers of $\Xi \rightarrow F(M \cap$ $U$ preserves $\left.A_{+}\right)$. The action of $G$ produces domains $\Xi(v), v \in V$, corresponding to other compact forms $X(v)$ of $Z$. In the example of the hyperboloid $Z_{1}$, if $X(0)$ is the standard real unit sphere, the domain $\Xi(0)$ in the cone is defined by the condition $\square(\xi)=\square(\eta)<1, \zeta=\xi+i \eta[\mathrm{Gi0} 4$.

The last element of the construction is the invariant holomorphic form $\omega(z ; d z)$ of the maximal degree on $Z$. It is defined up to a constant factor. On the compact manifolds $X(v)$ the restrictions of $\omega$ are the invariant measures and we can fix the normalization of $\omega$ such that all $X(v)$ have the unit full volume.

Horospherical Cauchy transform. Now we are ready to give the construction of the principal intertwining operator. Let us call the horospherical Cauchy kernel the meromorphic function

$$
K(z \mid \zeta)=\frac{1}{\prod_{1 \leq j \leq l}\left(1-\Delta_{j}(z \mid \zeta)\right)}, \quad \zeta \in \Xi, z \in Z
$$

The singular set of $K$ is the union of $l$ manifolds of the codimension 1 whose intersection is the horosphere $E(\zeta)$. Let $f \in \mathcal{O}(Z)$, we call its horospherical Cauchy transform the function

$$
\hat{f}(\zeta)=\int_{X(v)} K(z \mid \zeta) f(z) \omega(z ; d z) .
$$

If $\zeta \in \Xi(v)$ then be the Lemma the integrand has no singularities and defines a holomorphic function on $\Xi(v)$. These holomorphic functions give the holomorphic function on the whole manifold $\Xi$, since if $\zeta \in \Xi\left(v_{1}\right) \cap \Xi\left(v_{2}\right)$ then the value of $\hat{f}(\zeta)$ is the same for the integration on $X\left(v_{1}\right)$ and $X\left(v_{2}\right)$ since we integrate the closed form (holomorphic form of the maximal degree) on homological cycles and the parametric manifold $V$ is simply connected. Let us emphasize here a very natural structure of a singular integral operator on holomorphic functions where singular sets and 
cycles are disjoint and cycles of the integration are in the same homology class, but it is not sufficient to use one cycle for the global definition of the operator. Thus, we have defined an operator from $\mathcal{O}(Z)$ to $\mathcal{O}(\Xi)$.

It is possible to take the residue of the integrand-form on the horosphere $E(\Xi)$ but the cycles $X(v)$ can not be contracted to this horosphere, so we can not to reduce this integral to an integration on some cycles on the horosphere. This is not surprising since there are no nontrivial cycles on the horospheres.

Dual horospherical Cauchy transform. We want to inverse the horospherical Cauchy transform. Our inversion formula has a typical structure of Radon's type inversion formulas but transferred to the holomorphic setting. Firstly, we need to construct the dual intertwining operator from $\mathcal{O}(\Xi)$ to $\mathcal{O}(Z)$. Usually, such dual operators look very similar to direct operators. Such a possibility exists in our case, but since the complex geometry of $\Xi$ is different from the geometry of $Z$ we can realize another form of singular integral operators by a taking residue on singular sets; such form is simpler.

Let $\omega(\zeta, d \zeta)$ be the invariant holomorphic form of the maximal degree and $d a$ be the similar form on the Abelian group $A$. Instead of horospheres in the dual constructions we use dual submanifolds - pseudospheres $\Pi(z)$ in $\Xi$. The horospherical manifold $\Xi$ fibers on $A$-orbits of the dimension $l$ (over $F$ ). The pseudospheres $\Pi(z)$ are transversal to these fibers and they have complimentary dimensions $n-l$. Let us define the forms on $\Pi(z)$ as interior products

$$
\lambda(z, \zeta ; d \zeta)=d a\rfloor \omega(\zeta, d \zeta)
$$

They are forms $\phi$ such that $d a \wedge \phi=\omega$. The restrictions of such forms on $\Pi(z)$ are defined uniquely. The forms $\lambda(z, \zeta ; d \zeta)$ are holomorphic forms of the maximal degree on pseudospheres $\Pi(z)$. Such forms apriori are defined up to a factor dependent on $z$. The definition through the interior product fixes these factors up to a factor independent of $z$.

We call the dual horospherical Cauchy transform of $F \in \mathcal{O}(\Xi)$ the function

$$
\check{F}(z)=\int_{\Gamma(z)} F(\xi) \lambda(z, \xi ; d \xi), z \in Z
$$

Here the cycle $\Gamma(z)$ is any compact form of $\Pi(z)(\Pi(z)=(H \cap U) /(M \cap U))$. All these cycles (for a fixed $z$ ) are homological and their set is simply connected. The function $\check{F}$ is holomorphic.

We see that both horospherical transforms are connecting with some families of submanifolds: horospheres and pseudospheres correspondingly. On the pseudospheres (as opposed to the horospheres) there are appropriate non trivial cycles which we used for the definition of the dual horospherical transform. Nevertheless, it is possible to define the dual horospherical Cauchy transform also as a singular integral operator. There, as the integrand-form, we take the closed form $K(z \mid \zeta) \omega(\zeta, d \zeta)$ (as the holomorphic form of the maximal degree). As the cycles we take cycles

$$
\Gamma(z ; \varepsilon)=\{(\xi a) ; \xi \in \Gamma(z), a \in \Omega(\varepsilon), 1 \leq j \leq l\}
$$


where $\Omega(\varepsilon)$ is a cycle in $A$ which contracts to the unit element $e$ if $\varepsilon \rightarrow 0$. Consequently we consider some $\varepsilon$-tubular neighborhoods of cycles $\Gamma(z)$. We can define

$$
\check{F}(z)=c \int_{\Gamma(z ; \varepsilon)} F(\xi) \omega(\xi ; d \xi), z \in Z .
$$

In this definition the holomorphic dependence on $z$ is more explicit: for a small change of $z$ we can take homological cycles. To obtain the first presentation of the transform from the second one, we take the residue on $\Pi(z)$. It is a simple explicit computation since we have a product of $l$ singularities of 1 st order and the computation of the residue-form in each step reduces to the classical Cauchy formula.

The inversion formula. The last element of the consruction which we need for the inversion formula is a remarkable differential operator for a correction of the composition of the direct and dual horospherical transforms.

Let us consider invariant differential operators on the Abelian group $A$. We will characterize them by polynomial symbols $P(\mu)$ such that $P(D) a^{\mu}=P(\mu) a^{\mu}$. If $\mu=m_{1} \mu_{1}+\cdots+m_{l} \mu_{l}$, then $P$ is a polynomial on $m$. So in the natural sense these operators are operators with constant coefficients in logarithmical coordinates. Using the right action of $A$ on $\Xi$ we transfer the action of the operator $P(D)$ on functions $F(\zeta)$ on $\Xi$. Then

$$
P(D) \Delta^{\mu}(\zeta)=P(\mu) \Delta^{\mu}(\zeta), \Delta^{\mu}(\zeta)=\Delta_{1}^{m_{1}}(\zeta) \cdots \Delta_{l}^{m_{l}}(\zeta) .
$$

Let us call Weyl's operator the differential operator with the symbol

$$
W(\mu)=\prod_{\alpha \in \Sigma_{+}} \frac{\langle\mu+\rho, \alpha\rangle}{\langle\rho, \alpha\rangle},
$$

where $\rho$ is the half-sum of the positive roots. Hence the symbol is Weyl's formula for dimensions of irreducible finite-dimensional representations with the highest weight $\mu$.

Theorem $\mathbf{1}^{\prime}$. There is an inversion formula

$$
f=c(W(D) \hat{f}))^{\vee}, f \in \mathcal{O}(Z),
$$

where $c$ is a constant, depending on the normalization of the invariant differential forms. Moreover, the operators of horospherical Cauchy transform and the operator $F \mapsto c(W(D) F)^{\vee}$ are inverses of each other.

This Theorem is an explicit form of Theorem 1. Therefore we apply the Weyl operator to the horospherical Cauchy transform of $f$ and then take the dual horospherical Cauchy transform. This inversion formula has Radon's type structure. It shows that the composition of the dual and direct horospherical transforms is the Weyl differential operator on $\mathcal{O}(\Xi)$. The composition in the opposite order gives an invariant differential operator on $\mathcal{O}(Z)$.

If we were to write the operator in the inversion formula as the integral operator then we have a Cauchy-Fantappie type formula on $\mathcal{O}(Z)$ : it depends of choices of cycles $X(v)$ in $Z$ and $\Pi(z)$ in $\Xi$. 
Spherical Fourier transform. We have the decomposition of $\mathcal{O}(\Xi)$ in the Fourier series relative to the right action of the Abelian group $A$ :

$$
F(\zeta)=\sum_{\Lambda} F_{\mu}(\zeta), \quad F_{\mu}(\zeta \cdot a)=F_{\mu}(\zeta) a^{\mu}, \mu=m_{1} \mu_{1}+\cdots+m_{l} \mu_{l}, m_{j} \in \mathbb{Z}_{+} .
$$

It is the projection on irreducible representations of $G$. We call the spherical Fourier transform $\tilde{f}(\zeta ; \mu)$ the composition of the horospherical Cauchy transform with this Abelian Fourier transform:

$$
\hat{f}(\zeta)=\sum_{\mu \in \Lambda} \tilde{f}(\zeta ; \mu)
$$

We have

$$
\tilde{f}(\zeta ; \mu)=\int_{X(v)} f(z) \Delta^{\mu}(z \mid \zeta) \omega(z ; d z) .
$$

It is sufficient to remark that

$$
K(z \mid \zeta)=\sum_{\mu \in \Lambda} \Delta^{\mu}(z \mid \zeta), z \in X(v), \zeta \in \Xi(v), \mu=m_{1} \mu_{1}+\cdots+m_{l} \mu_{l} .
$$

We just decompose each factor in $K$ in the sum of the geometric sequence since $\left|\Delta_{j}(z \mid \zeta)\right|<1$ for $z \in X(v), \zeta \in \Xi(v)$. Then the direct integration on $X(v)$ gives this formula for $\zeta \in \Xi(v)$ and automatically for all $\zeta \in \Xi$ since the integrand is holomorphic on $\Xi$.

The direct application of the inversion formula for the horospherical transform to the presentation $\hat{f}$ through $\tilde{f}$ gives the inversion formula for the spherical Fourier transform (the Plancherel formula):

$$
f(z)=c \sum_{\mu \in \Lambda} W(\mu) \int_{\Gamma(z)} \tilde{f}(\zeta ; \mu) \lambda(z, \zeta ; d \zeta) .
$$

Hence we apply the dual horospherical Cauchy transform to the spherical Fourier transform (which is homogeneous relative to $A$ ). It is in a natural sense a complex analogue of the Poisson integral.

As an aside, one of the ways to prove the inversion formula for the horospherical transform is to prove in the beginning directly this inversion formula for the spherical Fourier transform: these 2 inversion formulas apparently are equivalent. There is a more conceptual way to prove the inversion formula by some tools of integral geometry (the method of the operator $\kappa$ ). We will publish these results in another paper.

Functions in subspaces $\left.\mathcal{O}_{\mu}(\Xi)\right)$ are joint eigen functions for the invariant differential operators $P(D)$ (with the differentiations only along $A$-fibers). The dual horospherical Cauchy transform transforms these irreducible horospherical $G$-modules in equivalent spherical $G$-modules on $Z$. Functions in these subspaces are also joint eigen functions of invariant differential operators on $\mathcal{O}(Z)$. The horospherical Cauchy transform connects invariant differential operators on $\mathcal{O}(Z)$ with the operators $P(D)$ on $\mathcal{O}(\Xi)$ with differentiations only along $A$-fibers. 
Some remarks. I believe that it is interesting to discuss these results from point of view the point of view of representations theory as well as from the point of view of complex analysis. Here are a few remarks.

1. From the point of view of representations we observe a rare example of equivalency of non unitary infinite dimensional representations of different origins. It is essential sometimes to work directly with such representations. We need to better to understand the phenomena of series of representations and their models. Irreducible representations sometimes appear in decompositions of some interesting representations not individually, but by complete series (as for cusps of fundamental domains of discrete subgroups). For such situations it is important to have models of such series (not necessary for the continuous spectrum). We consider here spherical and horospherical models of series of finite dimensional representations.

It is interesting to find other examples of such non trivial equivalences. Probably such dual objects exist for some nonsymmetric Stein homogeneous manifolds with semisimple Lie groups.

2. From the point of view of complex analysis we have an unusual example of an explicit isomorphism of spaces of holomorphic functions which is neither a corollary of biholomorphic equivalency nor a consequence of some constructions of functional analysis. The relation between $\mathcal{O}(Z)$ and $\mathcal{O}(\Xi)$ is rather a duality relation (we present holomorphic functions as some analytic functionals). There are several important dualities in complex analysis. One example is Martineau's duality for linear concave domains. There we have duality between some cohomology (analytic functionals) and holomorphic functions, but I do not know other non trivial examples of dualities between two spaces of holomorphic functions.

3. In the focus of our considerations is the conception of singular integral operators on holomorphic functions. I believe that such general structure of families of meromorphic kernels and totally real cycles which are disjoint with singular sets looks very natural, but it is not simple to satisfy these geometrical conditions of the separation of singularities of kernels and cycles. I do not think that it is simple to produce nontrivial examples without some exterior tools (as groups in our example). I know only one more example where similar geometrical situation appears: Cauchy formula on compact Hermitian symmetric spaces for different realizations of dual spaces. The construction with residues is simpler, but also reflects a non trivial geometrical situation.

4. All our constructions can be described purely geometrically with dual families of submanifolds and some real cycles: we used groups only for proofs. It would be interesting to find some conditions where the analogue of horospherical transform admits a similar inversion (with a dual transform and a differential operator). It would be a combined problem of nonlinear analysis and integral geometry.

Unitary restriction. The principal point of the theory of finite-dimensional representations of complex semisimple and compact Lie groups is that these 2 theories essentially coincide: irreducible representations are the same (unitary trick of Weyl). One of the explanations of this phenomenon is that spherical finitedimensional representations of compact Lie groups are realized in eigenspaces of invariant differential operators on these groups; these operators are elliptic and all 
eigenfunctions simultaneously holomorphically extend on the complexifications of the groups. If we were to work with reducible representations in the direction which we developed above, the cases of compact and complex symmetric spaces would be quite different. It is similar to the fact that the theories of entire functions and functions on the circle are quite different in spite of the connection between Taylor and Fourier series.

A first observation is that if instead of holomorphic functions on $Z$ we were to consider $C^{\infty}$ - functions on the compact symmetric space $X(0)$, the formulas above survive. More precisely, if $f \in C^{\infty}(X(0))$ then its horospherical Cauchy transform $\hat{f}(\zeta)$ is well-defined and holomorphic for $\zeta \in \Xi(0)$. Under these condition $\hat{f}$ has $C^{\infty}$ boundary values on the boundary $\partial \Xi(0)$. For any $z \in X(0)$ the pseudosphere $\Pi(z)$ intersects $\partial \Xi(0)$ exactly on one cycle $\Gamma(z) \cong U / M$. So the expression for the inversion formula makes sense (but loses the freedom in the choice of the cycle $\Gamma(z)$, as compared with the $Z$-case) and it turns out that the inversion holds in this case as well. Moreover, the proof of the inversion formula by tools of integral geometry has no holomorphic essentials and directly applies to this case.

The definition of the horospherical transform and its inversion do not need such strong conditions on $f$ as $C^{\infty}$. The definition can be easy extended on locally integrable functions and distributions. We will focus on the case of hyperfunctions and problems of complex analysis in the process of these considerations.

Theorem 2. There is an isomorphism of $U$-modules

$$
\operatorname{Hyp}(X(0))=H^{(n-l)}(Z \backslash X(0), \mathcal{O})=\mathcal{O}(\Xi(0)) .
$$

Let us explain all objects in this statement and construct the intertwining operators. We define hyperfunctions as analytic functionals on the space of holomorphic functions on the compact $X(0)$ :

$$
\operatorname{Hyp}(X(0))=(\mathcal{O}(X(0)))^{\prime} .
$$

We imbed $\mathcal{O}(Z)$ in $\operatorname{Hyp}(X(0))$ :

$$
\Phi_{f}(\phi)=\int_{X(0)} f(z) \phi(z) \omega(z ; d z), \quad f \in \mathcal{O}(Z), \phi \in \mathcal{O}(X(0)) .
$$

Let us call the horospherical Cauchy transform of the hyperfunction $\Phi$ the holomorphic function

$$
\hat{\Phi}(\zeta)=\Phi(K(\cdot \mid \zeta)), \quad \zeta \in \Xi(0) .
$$

We used here the fact that for any $\zeta \in \Xi(0)$ the kernel Cauchy $K(\cdot \mid \zeta)$ as a function of $z$ lies in $\mathcal{O}(X(0))$. Apparently, this definition of the horospherical Cauchy transform of hyperfunctions is compatible with the definition of the horospherical transform of holomorphic functions on $Z$.

We will use the holomorphic language for analytic cohomology [EGW95]. Specifically, we consider the manifold

$$
Y(0)=\{(z, \zeta) ; z \in(Z \backslash X(0)), \zeta \in \Xi(0), z \in E(\zeta)\} .
$$


Thus we take pairs of points on $Z$ outside of $X(0)$ and passing through them horospheres which do not intersect $X(0)$. It is a Stein manifold which has natural fiberings over $Z \backslash X(0)$ and $\Xi(0)$ (double fibering). It is essential that fibers of first fibering are contractible.

We consider the complex of holomorphic forms on $Y(0)$ with differentials only on $\zeta: \psi(z, \zeta, d \zeta)$. The differential also acts only on $\zeta$. As proved in [EGW95] under conditions which are satisfied in this example cohomology of this complex are isomorphic to Dolbeault cohomology. We will see that this language is very convenient for our situation.

Now we will realize the cohomology as hyperfunctions. We need a geometrical preparation. The edge $\Theta$ of the boundary of $\Xi(0)$ is a compact of the real dimension $2 n-l$. In the fibering $\Xi$ over $F$ the cycle $\Theta$ has as fibers the torus $T$. The right action of $T$ preserves $\Xi(0)$ and $\Theta$. The semigroup $A_{+}$acts on $\Xi(0)$ by contractions and we can consider homothetic images $\Theta_{\rho}$ of $\Theta$ inside $\Xi(0)$.

On the other side, $\Theta$ is fibered over $X(0)$ on the cycles $\Gamma(z), z \in X(0)$. In such a way we can identify $\Theta$ with the edge $\tilde{\Theta}$ of the boundary of $Y(0)$. We see that the real dimension of $\tilde{\Theta}$ coincides with the complex dimension of $Y(0)$. Thus it has the minimal possible dimension. Let cycles $\tilde{\Theta}_{\rho}$ be any liftings of $\Theta_{\rho}$ in $Y(0)$ (they are not defined uniquely by the difference with $\tilde{\Theta})$.

Let $\psi(z, \zeta ; d \zeta)$ be a holomorphic closed (on $\zeta)(n-l)$-form on $Y(0)$ with the differentials only on $\zeta$. We define the hyperfunction as

$$
\Phi_{\psi}(\phi)=\int_{\tilde{\Theta}_{\rho}} f(z) \psi(z, \zeta ; d \zeta) \wedge \omega(z ; d z), f \in \mathcal{O}(X(0))
$$

where the cycle $\tilde{\Theta}_{\rho}$ is constructed above the cycle such that it lies in the holomorphy domain of the function $f$. It turns out that this is an isomorphism between hyperfunctions and cohomology. We define the horospherical Cauchy transform as

$$
\hat{\psi}(\zeta)=\hat{\Phi}_{\psi}(\zeta), \quad \zeta \in \Xi(0)
$$

This definition depends only on the cohomology class and we have the horospherical Cauchy transform for $H^{(n-l)}(Z \backslash X(0), \mathcal{O})$.

Now we define the dual horospherical Cauchy transform of $F \in \mathcal{O}(\Xi(0))$. It is the cohomology class of the form

$$
\check{F}=F(\zeta) \lambda(z, \zeta ; d \zeta)
$$

The analogue of Theorem $1^{\prime}$ holds for the constructed horospherical transform of cohomology.

Theorem $2^{\prime}$. 1)If $\psi$ is a holomorphic $d_{\zeta^{-}}$closed $(n-l)$-form on $Y(0)$ then

$$
c(W(D)(\hat{\psi}))^{\vee}-\psi
$$

is $d_{\zeta}$-exact. 
2)For $F \in \mathcal{O}(\Xi(0))$ we have

$$
F(\zeta)=c\left((W(D) F)^{\vee}\right)^{\wedge}
$$

Let us emphasize that we take in Theorem the horospherical Cauchy transform from holomorphic forms to holomorphic functions and the dual horospherical Cauchy transform transforms holomorphic functions in forms. In this inversion formula we construct through the horospherical Cauchy transform $\hat{\psi}$ of a form $\psi$ a form such that it lies in the same cohomology class as $\psi$. So we reconstruct cohomology classes through their horospherical Cauchy transforms.

The last relation is the Cauchy type formula in $\Xi(0)$ :

$$
F(\zeta)=c \int_{\tilde{\Theta}_{t}} K(z \mid \zeta) W(D)(F(\zeta)) \lambda(z, \zeta ; d \zeta) \omega(z ; d z), f \in \mathcal{O}(\xi(0)), \zeta \in \Xi(0), t \in A_{+}
$$

The injectivity of the horospherical Cauchy transform on cohomology is also some fact of complex analysis about a solvability of $d_{\zeta}$-equations. Another important fact is the possibility to reconstruct an analytic functional (hyperfunction) through its evaluations on holomorphic functions $K(\cdot \mid \zeta)$. It is a consequence of Martineau's type integral formula for holomorphic functions in some neighborhoods of $X(0)$.

We need to connect Theorems 1 and2 : for holomorphic functions on $Z$ and for hyperfunctions on $X(0)$. For this aim let us discuss a possibility to define boundary values on $X(0)$ of cohomology in $H^{(n-l)}(Z \backslash X(0), \mathcal{O})$ as functions on $X(0)$. Let $\psi(z, \zeta ; d \zeta),(z, \zeta) \in Y(0)$, be a $d_{\zeta}$-closed form from a cohomology class. The edge of the boundary of $Y(0)$ is identified with the edge $\Theta$ of the boundary of $\Xi(0)$ which is fibering over $X(0)$ with the fibers $\Gamma(z)$. Let a holomorphic form $\psi$ has boundary values in some sense on $\Theta$. Then we can integrate the boundary form on cycles $\Gamma(z)$ and the resulting function $b_{\psi}(z)$ on $X(0)$ can be interpreted as the boundary function for the cohomology class. Of course such functions exist only for some special cohomology classes, but if a form $\psi$ has holomorphic or continuous extension on $\Theta$ we can realize this construction.

If we have a locally integrable function $f$ on $X(0)$, we explained how to construct the corresponding hyperfunction $\Phi_{f}$. We take its horospherical transform $\hat{\Phi}_{f}$ and its inversion as in Theorem $2^{\prime}$ gives an explicit differential form $\psi(z, \zeta ; d \zeta),(z, \zeta) \in$ $Y(0)$, in the same class as $\Phi_{f}$. If there exist the boundary function $b_{\psi}$ we can compare it with $f$. The direct consideration of the inversion formula for $f \in \mathcal{O}(Z)$ shows that in this case $f=b_{\psi}$. It generalized automatically for $f \in \mathcal{O}(X(0))$ and, after simple considerations, for $f \in C^{\infty}(X(0)$. The case of distributions $f$ slightly more complicated.

Corollary. In each cohomology class $H^{(n-l)}(Z \backslash X(0), \mathcal{O})$ in the holomorphic description there is one and only one representative of the form $F(\zeta) \lambda(z, \zeta ; d \zeta)$.

To construct such a form we can start from any form in the cohomology class, to make the horospherical transform. Its inversion in our formula will be such canonical representative in the cohomology class. This corollary can be interpreted as a 
holomorphic version of the Hodge Theorem. Hodge's type theorems give a canonical choice of representatives in cohomology classes using some additional structures, usually some metrics. In our construction we select canonical representatives using a complex geometry (other examples see in [GH78, EGW95]).

Some remarks. 1. Most important consequence of these considerations is that apparently compact symmetric spaces $X(0)$, including compact Lie groups, have canonical dual objects - complex manifolds $\Xi(0)$ which are domains in horospherical manifolds $\Xi$. These domain are $U$-invariant, but they are not homogenous.

2. Since the domain $\Xi(0)$ is invariant relative to right action of semigroup $A_{+} \supset T$ we can decompose $\mathcal{O}(\Xi(0)$ relative to this action. The components of this decomposition will coincide with $\mathcal{O}_{\mu}(\Xi)$ since $A_{+}$-homogenous holomorphic functions on $\Xi(0)$ can extend on $\Xi$ (holomorphic characters of $A_{+}$and $A$ are the same); this is just the unitary trick. However, if not restricted by irreducible representations, the analysis on $X(0)$ does not reduce to the analysis on $Z$. We can investigate the decomposition of hyperfunctions on compact symmetric spaces on spherical functions.

3.We worked with holomorphic language of analytic cohomology. Let us recall that there is a very simple way to transfer to Dolbeault cohomology [GH78,EFG95]. We need to restrict a holomorphic form $\psi$ in $Y(0)$ on any $C^{\infty}$-section of the fibering $Y(0) \rightarrow(Z \backslash X(0))$, to consider this restriction as a form on the base and to take its $(0, n-l)$-part.

4. It is natural to compare the horospherical transform with the Penrose transform which associates analytical cohomology by integration along complex cycles with some holomorphic functions. In our case the manifold $Z \backslash X(0)$ has no complex cycles at all. We follow to another classical conception - Fantappie's indicators. Namely, we realize analytic cohomology as analytic functionals and consider their evaluations on a family of holomorphic functions dependent on complex parameters $K(\cdot \mid \zeta)$. Our considerations around Theorem $2^{\prime}$ are very similar to Martineau results on complex concave domains: horospheres replace hyperplanes in his construction (cf.also [GH78]).

5.Let us remark that in a sense $\mathcal{O}(Z)$ can be considered as the intersection of $H^{(n-l)}(Z \backslash X(v), \mathcal{O}), v \in V$.

6 .These considerations give several interesting examples of global explicit constructions in multidimensional complex analysis on the basis of an explicit geometry. Let us note that we connect hyperfunctions on $X(0)$ with cohomology of codimension $l$, not 1 as in classical examples.

7. One of the most interesting development of this constructions is special functions on $Z, \Xi$. The basic tools are "elementary" functions $\Delta^{\mu}, K$ and cycles $X(v), \Theta$. The possibility to deform contours in the complex domain is a most exciting challenge. Integral representations of special functions on homogeneous manifolds so far remind me of real integral representations of classical special functions before the application of the complex contour integration. Such a take on $c$-function of Harish-Chandra is one of the first problems.

8. The basic direction of this project is the consideration of pseudo Riemann forms of $Z$. We consider the real forms $Z_{\mathbb{R}}=G_{\mathbb{R}} / H_{\mathbb{R}}$ where the real form $G_{\mathbb{R}}$ corre- 
sponds to an involution which commutes with the involution for $H$. The difficulty is that $Z_{\mathbb{R}}$ is not a cycle if it is not a compact symmetric space. The conjecture is that nevertheless the inversion formula survives in this case as well. There are several examples of such formulas [Gi00, Gi02,Gi04, Gi05', GKO05] which show that cycles $\Gamma(z)$ can be quite complicated (to have several components, corresponding to different series of representations) or can stop being cycles. It is connected with an understanding of the analytic nature of different series and develops our old project [GG77].

\section{REFERENCES}

[Cl88] J.-L.Clerc, Functions sphériques des espaces symétriques compacts, Trans.AMS 306 (1988), 421-431.

[EFG95] M.Eastwood,S.Gindikin, H.-W.Wong, Holomorphic realization of $\bar{\partial}$-cohomology and constructions of representations, J.Geometry and Physics 17 (1995), 231-244.

[GG77] S. Gindikin, I.Gelfand, Complex manifolds whose skeletons are real semisimple Lie groups and holomorphic discrete series of representations, Funct.Anal. Appl. 11:4 (1977), 19-27.

[Gi00] S.Gindikin, Integral Geometry on $S L(2 ; \mathbb{R})$, Math.Res.Letters 7 (2000), 417-432.

[Gi00'] S.Gindikin, Integral geometry on hyperbolic spaces, Harmonic Analysis and Integral Geometry, (M.Picardello, ed.), Chapman and Hall, 2000, pp. 41-46.

[Gi02] S. Gindikin, An analytic separation of series of representations for $S L(2 ; \mathbb{R})$, Moscow Math. J. 2 (2002), no. 4, 1-11.

[Gi04] S.Gindikin, Complex horospherical transform on real sphere, geometric Analysis of PDE and Several Complex Variables. Contemporary Mathematics, vol. 368, Amer. Math. Soc., 2005, pp. 227-232.

[Gi05] S.Gindikin, Horospherical Cauchy-Radon transform on compact symmetric spaces, preprint, ArXiv math./RT/0501022 (2004).

[Gi05'] S.Gindikin, Holomorphic horospherical duality "sphere-cone", preprint, ArXiv mathCV/0501021 (2005) (to appear Indagationes Mathematicae).

[GH78] S.Gindikin and G.Henkin, Integral geometry for $\bar{\partial}$-cohomology in q-linearly concave domains in $\mathbb{C} P^{n}$, Funct.Anal.Appl. 12 (1978), no. 4, 6-23. (Russian)

[GH90] S.Gindikin and G.Henkin, The Cauchy-Fantappie on projective space, Amer. Math. Soc. Transl.(2) 146 (1990), 23-32.

[GKO05] S. Gindikin, B. Krötz and G.Ólafsson, Horospherical model for holomorphic discrete series and horospherical Cauchy transform, preprint, ArXiv math.RT/0411564 (2005).

[H94] S.Helgasson, Geometric analysis on symmetric spaces, AMS, 1994.

Departm. of Math., Hill Center, Rutgers University, 110 Frelinghysen Road, Piscataway, NJ 08854-8019

E-mail address: gindikin@math.rutgers.edu 